\title{
INSERÇÃO PROFISSIONAL DA/O PSICÓLOGA/O ESCOLAR EM INSTITUIÇÕES PÚBLICAS DO PIAUÍ: GEORREFERENCIAMENTO E POLÍTICAS EDUCACIONAIS
}

\author{
PROF. DR. FAUSTON NEGREIROS* \\ RAFAELA BRENDA ARAÚJO DA SILVA** \\ JANAINA OLIVEIRA ROCHA*** \\ THAISA DA SILVA FONSECA**** \\ LEILANIR DE SOUSA CARVALHO***** \\ FERNANDA MARIA DE OLIVEIRA******
}

\begin{abstract}
RESUMO
Este trabalho foi desenvolvido com o objetivo de identificar e georreferenciar a inserção da/o psicóloga/o escolar em instituições públicas de ensino - de âmbito municipal, estadual e federal - junto às políticas públicas educacionais no estado do
\end{abstract}

* Professor associado da Universidade Federal do Piauí/UFPI, do Departamento de Psicologia e do Programa de Pós-Graduação em Psicologia. Pós-Doutorado em Psicologia Escolar e do Desenvolvimento Humano pela Universidade de São Paulo USP. Coordenador do PSIQUED. Membro do GT Psicologia e Política Educacional da ANPEPP. Orientador da pesquisa.

** Graduanda em Psicologia pela Universidade Federal do Piauí / UFPI. Iniciação Científica em Pesquisa do Núcleo de Pesquisas e Estudos em Desenvolvimento Humano, em Psicologia Educacional e Queixa Escolar/PSIQUED. Membra do Núcleo Piauí do Fórum sobre a Medicalização da Educação e da Sociedade.

*** Psicóloga graduada pela Universidade Federal do Piauí-UFPI/ Campus Ministro Reis Velloso, mestranda do Programa de Pós-Graduação em Psicologia-PPGPsi/ UFPI, membra do Núcleo de Pesquisas e Estudos em Desenvolvimento Humano, Psicologia Educacional e Queixa Escolar-PSIQUED.

**** Psicóloga, mestra em Psicologia e membra do Núcleo de Pesquisa e Estudos em Desenvolvimento Humano, Psicologia Educacional e Queixa Escolar - PSIQUED/UFPI.

***** Psicóloga, mestra em Psicologia e membra do Núcleo de Pesquisa e Estudos em Desenvolvimento Humano, Psicologia Educacional e Queixa Escolar - PSIQUED/ UFPI.

****** Psicóloga do Núcleo de Apoio Estudantil/NAE da UFPI. Mestra em Psicologia pelo Programa de Pós-Graduação em Psicologia-PPGPsi/UFPI, membra do Núcleo de Pesquisas e Estudos em Desenvolvimento Humano, Psicologia Educacional e Queixa Escolar-PSIQUED. 
Piauí. Tratou-se de uma pesquisa de abordagem qualitativa. Para a coleta de dados foram utilizados como instrumentos: questionário sociodemográfico, formulário de análise documental, formulário de políticas públicas e roteiro de entrevistas. A pesquisa contou com a participação de 72 psicólogas/os atuantes em instituições públicas de ensino. O perfil do participante compôs-se predominantemente por profissionais do sexo feminino (84,7\%) com média de idade de 35-59 anos, distribuídas/os em 21 municípios piauienses, sendo a maioria atuante na capital do estado, Teresina. Em relação à atuação das/os psicólogas/os em políticas públicas educacionais, os resultados apontam para a inserção nos seguintes perfis de políticas: Políticas de Melhoramento da Educação (60,9\%), Políticas para a Educação Inclusiva (15,6\%), Políticas de Formação de Professores (9,4\%), Políticas Intersetoriais (6,3\%), Políticas de Formação e Atuação da/o Psicóloga/o Escolar (4,7\%), Políticas de Atendimento à Diversidade (3,1\%). Em suma, pôde-se identificar a massiva inserção da/do profissional da psicologia escolar nas regiões mais abastadas e próximas à capital do referido estado, bem como a sua maior articulação com as políticas que objetivam a melhoria da qualidade da educação, tanto em relação ao acesso e permanência, quanto ao enfrentamento de problemas no processo de escolarização.

Palavras-chave: Psicologia Escolar. Políticas Educacionais. Rede Pública de Ensino.

\begin{abstract}
This work was developed with the aim of identifying and georeferencing the insertion of the school psychologist in public schools - of municipal, state and federal contexts - along with public educational policies in the state of Piauí. It was a qualitative research and, for data collection, sociodemographic questionnaire, document analysis form, public policy form and interview script instruments were used. The research had the participation of 72 psychologists working in public educational institutions. The participant profile was predominantly presented
\end{abstract}


by female professionals (84.7\%) with an average age of 35.59 years, distributed in 21 municipalities in Piauí, the majority of whom are active in the state capital, Teresina. Regarding the performance of psychologists in public educational policies, the results pointed to the performance in the following policy profiles: Education Improvement Policies (60.9\%), Policies for Inclusive Education (15.6\%), Training Policies of Teachers (9.4\%), Intersectoral Policies (6.3\%), Policies for Training and Performance of the Educational Psychologist (4.7\%), Policies for Assistance to Diversity (3.1\%). In short, the massive insertion of school psychology professionals in the wealthier regions and close to the capital of that state can be identified, as well as their greater articulation with policies aimed at improving the quality of education, both in relation to access and permanence, in relation to facing problems in the schooling process.

Keywords: School Psychology. Educational Policies. Public School.

\section{INTRODUÇÃO}

A partir do século XVI os ideais liberalistas ganham força na sociedade, assim, passa-se a defender a mínima intervenção do Estado na economia, utilizando o mercado como o regulador natural das relações sociais, ou seja, o valor do indivíduo e de sua relação passa a ser percebido mediante sua inserção no mercado produtivo. No entanto, tal organização colapsa resultando na maior crise econômica em 1929 produzindo impactos negativos na sociedade em todo o mundo. Esse panorama antecedeu a deflagração da segunda guerra mundial, da guerra fria e de vários outros conflitos ideológicos, políticos e econômicos. (CRUZ; GUARESCHI, 2017).

É diante dessa atmosfera social de adversidades, injustiças e violência do período pós-guerras que as políticas públicas ganham forma, pois a configuração social, política e econômica passou a demandar uma interferência mais incisiva do Estado com o objetivo de garantir o bem-estar social, isto é, as políticas 
públicas surgem da necessidade de cuidado social. Neste aspecto, em linhas gerais, pode-se perceber que desde o princípio, as políticas públicas são formuladas em resposta a possíveis demandas ou tensões que surgem na sociedade, ou seja, são pensadas a partir de uma necessidade de proteção social (CRUZ; GUARESCHI, 2017; LEONARDO; ROSSATO; CONSTANTINO, 2018).

Ainda segundo ressalta Dimenstein (2011, p. 119), mesmo que discussões envolvendo políticas públicas sejam em geral complexas, é importante compreender que "todos nós somos operadores de políticas, mesmo lá nos espaços mais recônditos da vida privada".

Mediante as reflexões supracitadas acerca do contexto histórico de surgimento das políticas públicas pode-se compreendê-las como princípios que norteiam a ação do poder público e diretrizes para as relações estabelecidas entre poder público e sociedade, ou seja, regulamentam interações no coletivo e podem se institucionalizar por meio de programas, leis, ações, projetos, entre outros. (DIMENSTEIN, 2011; FERNANDES; MÉLO, 2016; MORAES; CARVALHO; COSTA; NEGREIROS; FREIRE, 2019).

No que se refere ao Brasil, a promulgação da Constituição Federal de 1988 foi um marco para a luta e garantia de direitos, pois nesse contexto passa a ser dever do Estado se responsabilizar pelas questões sociais e políticas de educação. (FARAH, 2016). Dessa forma, as políticas educacionais especificamente se apoiam no dever do Estado de garantir o direito à educação a todo cidadão.

Entretanto, ainda que na Constituição a educação enquanto direito fundamental tenha surgido amparada na necessidade de justiça social, uma vez que ela representaria uma possibilidade de ascensão social, é importante destacar que esse direito também respondia à demanda do capitalismo por trabalhadores qualificados. (LESSA; TONET, 2013; SANTOS, 2017). Nesse cenário, os conhecimentos da Psicologia, enquanto ciência, e o próprio 
psicólogo passaram a ser requeridos nas áreas da assistência social, educação e saúde, evidenciado por transformações no fazer cientifico da Psicologia. (MORAES ET AL, 2019).

Além disso, é importante atentar-se para o contexto social do estabelecimento do capitalismo enquanto modelo sociopolítico e econômico, no qual a Psicologia se dissemina. Isso contribui para que se entenda a forma como essa ciência se colocou a serviço da regulação e controle da população, ou seja, um saber popularizado como ferramenta de controle social necessário para a manutenção do sistema político e econômico. (SILVA; CARVALHAES, 2016).

Segundo Foucault (2010), o saber psi teve um papel fundamental no processo de normatização dos estilos e controle da vida, com o intuito de adequar as pessoas ao ditos comportamentos normais, muitas vezes pela via da criminalização ou psicologização de tudo aquilo que se situava fora deste nicho padronizado. Essa orientação produz conhecimentos e práticas que permeiam famílias e instituições escolares, desempenhando um papel normativo e medicalizante. (MANSANO, 2009).

No decorrer do século XX, dentro de uma racionalidade capitalista, pode-se salientar a importância das ciências sociais para o desenvolvimento da sociedade industrial e de consumo, que assumiram uma responsabilidade no campo da produção científica como saber fundamental na consolidação da organização social na modernidade. Com esse posicionamento, as práticas coletivas e individuais passaram a ser problematizadas em áreas como a Antropologia, Economia, Política e própria Psicologia. E na atualidade, é possível perceber reverberações dessas produções em intervenções psicológicas no campo das políticas públicas no Brasil, que, por vezes, permanecem com o intuito de ditar o ritmo de vida no cotidiano. Em contrapartida, há a atuação com práticas psicológicas críticas às formas de normalização social, como, por exemplo, o reconhecimento de diferentes dinâmicas familiares, valorização de expressões culturais periféricas, etc. (ROMAGNOLI, 2006). 
Portanto, a história da Psicologia se expressa mediante as diferentes formas de controle e de resistências que transitaram ao longo da sociedade. E isso ainda se reflete nas últimas décadas, uma vez que, mesmo ocupando novos espaços de intervenção, por vezes, observa-se a dificuldade que tais profissionais ainda enfrentam entre continuar seguindo modelos tradicionais e passar a construir modelos críticos de atuação.

Somente com a reforma democrática do Estado brasileiro, a partir da década de 1990, houve uma liberdade no âmbito educacional para pensar em efetivas políticas de educação, e nesse ponto as relações entre Psicologia, políticas e educação são aproximadas e ganham relevância. (SOUZA; CUNHA, 2010). Segundo Zibetti, Pacífico e Tamboril (2018), a atuação da Psicologia junto às políticas públicas educacionais, assim como discutido anteriormente, esteve apoiada em conhecimentos convenientes para o ideal de diminuição das diferenças e controle dos modos de ser e agir dos sujeitos.

Neste aspecto, Souza et al (2014) ressaltam que o atual contexto educacional no Brasil requer que a atuação profissional do Psicólogo Escolar e Educacional (PEE) esteja comprometida com questões que vão além dos processos estritamente psicológicos, mas que esteja transversalmente aliada a conhecimentos políticos e sociais tendo em vista que estes produzem todo o contexto escolar e as relações que o permeiam. Destarte, segundo Collares-da-Rocha e Oliveira (2020), a atuação política do psicólogo escolar não se restringe aos muros da escola e viabiliza a promoção de cidadania e defesa dos direitos humanos, a participação como figura ativa no processo de construção e operacionalização das políticas públicas de educação.

Nesse sentido, de acordo com Guzzo, Costa e Sant'Ana (2009), faz-se necessário que o PEE promova reflexões acerca de sua atuação constantemente em busca de corresponder às necessidades da escola e da comunidade. Desse modo, Damasceno e Negreiros (2018) pontuam a importância de a/o profis- 
sional familiarizar-se quanto às particularidades da comunidade escolar com a qual trabalha, assim como com as potencialidades e possíveis dificuldades presentes, a fim de que possa planejar ações relacionadas a essa realidade específica com o objetivo de contribuir com o processo de escolarização.

Em se tratando da psicologia no contexto piauiense, Silva e Yamamoto (2013) evidenciam a recente implementação de cursos de graduação na área, se comparado à realidade de outros estados brasileiros. Essa implantação foi impulsionada pela política de expansão da educação superior operacionalizada por meio da Lei de Diretrizes e Bases da Educação Nacional (Lei n ${ }^{\circ}$ 9.394, 1996). Expansão que viabilizou o crescimento da psicologia no estado do Piauí, tanto no que diz respeito à quantidade de profissionais cadastrados, quanto em relação à produção científica nessa área na realidade piauiense.

Por outro lado, ainda há uma variedade de limitações para atuação e pesquisa em Psicologia e Políticas Públicas no Piauí. As dificuldades estão em geral associadas ao processo formativo da maioria dos cursos, com diretrizes curriculares pouco favoráveis para aquisição de conhecimento e experiências para além da atuação clinica tradicional, desarticulação entre teoria e prática, entre outras. (SILVA; NEGREIROS, 2008; CARVALHO, 2007).

A pesquisa realizada por Macedo e Dimenstein (2011) oferece resultados que apontam para a relevância de se investigar a forma como está sendo desenhada a prática da Psicologia em Políticas Públicas, haja vista que, de acordo com o estudo, $47,28 \%$ dos profissionais com cadastro no Conselho Regional de Psicologia atuam nos dispositivos públicos do estado. Este cenário pode servir como ponto de reflexão para analisar a inserção desse profissional junto às políticas públicas de educação na região Nordeste do Brasil, que segue em desenvolvimento gradual, como sinalizam Negreiros e Campos (2020).

Percebe-se nas últimas décadas, o aumento do número de profissionais psicólogos nos campos articulados às políticas 
públicas. A pluralidade com que o trabalho se engendra nestes espaços aponta para a importância de se analisar algumas particularidades e possibilidades que produzem as atuações das psicólogas e psicólogos nas políticas públicas de educação.

Dessa maneira, este estudo tem como ponto de partida o seguinte objetivo: identificar e georreferenciar a inserção da/o psicóloga/o escolar em instituições públicas de ensino - de âmbito municipal, estadual e federal - junto às políticas públicas educacionais no estado do Piauí, identificando, essencialmente, aquelas nas quais esses profissionais se inserem no desempenho de seu trabalho.

\section{METODOLOGIA}

A escolha teórico-metodológica deste estudo fundamenta-se na abordagem qualitativa. Este método objetiva captar determinado fenômeno a partir da visão das pessoas nele implicadas, envolvendo, com este fim, o estudo das relações, estruturas sociais, representações, hábitos, opiniões, etc. (TURATO, 2005). Neste aspecto, a pesquisa se especifica como descritiva, uma vez que se propõe a descrever fenômenos de determinada realidade (GIL, 2010).

\section{PARTICIPANTES}

Para o desenvolvimento da referida pesquisa utilizou-se como fonte o banco de dados composto por informações coletadas nos últimos quatro anos, do projeto intitulado "Atuação do Psicólogo Escolar em Políticas Públicas de Educação no Piaui”, que possui as seguintes ramificações: Banco EB (composto por Psicólogos Escolares da Educação Básica do Estado do Piau1), coordenado por Leilanir de Sousa Carvalho; Banco IF (integrado por Psicólogos dos IFPIs), coordenado por Thaisa da Silva Fonseca; e o Banco UF (formado por Psicólogos atuantes em campis da UFPI), coordenado por Fernanda Maria de Oliveira.

É importante destacar que esse banco de dados está sob responsabilidade do Núcleo de Pesquisas e Estudos em Desen- 
volvimento Humano, Psicologia Educacional e Queixa Escolar/ PSIQUED, sob a coordenação geral do professor Fauston Negreiros, orientador da produção dos aludidos bancos de dados e pesquisas.

As/os participantes somaram um total de 72 psicólogas e psicólogos. Dessas, 42 atuam na rede pública de educação do estado do Piauí, sendo que 20 estão vinculadas aos Institutos Federais do Piauí (IFPIs) e 10 distribuídas em instituições públicas de Ensino Superior. A maior parte dos participantes da pesquisa é do sexo feminino $(84,7 \%)$ e com média de idade de 35-59 anos.

Quanto à cidade em que exercem sua função, uma quantidade expressiva de psicólogas se concentra na capital do estado, Teresina (38,8\%), seguidas por 11,1\% que atuam em Parnaíba. Os demais estão distribuídos em: Floriano, Picos e Paulistana com 4,2\% cada uma. Já os municípios de Bom Jesus, Corrente, Oeiras, Piripiri, São João do Piauí, São Raimundo Nonato, Uruçuí e Valença englobam 2,7\% dos participantes em cada cidade. Altos, Angical, Barras, Caldeirão Grande do Piauí, Campo Maior, Cocal, Cocal dos Alves, Fronteiras, Pedro II, Regeneração e União, contam com 1 psicólogo por cidade.

Com relação à vinculação profissional das psicólogas e psicólogos que estão inseridos na Educação Básica, a maioria atua na Secretaria de Estado de Educação/SEDUC do Piauí. Um total de 40 participantes está distribuído entre Gerências Regionais de Educação/GREs do estado, Coordenação do Pronatec, Coordenação do Núcleo de Atenção Psicossocial/NUAPSI e na própria Seduc (não locados em nenhum setor específico do órgão). E as outras duas participantes atuam na Secretaria Municipal de Educação/SEMEC de Teresina.

Acerca dos participantes vinculados ao IFPI, estes estão lotados predominantemente no setor de saúde (73,3\%), seguidos por $20 \%$ que trabalham por meio da Diretoria de Ensino/Departamento Pedagógico, enquanto 6,7\% atuam na Pró-Reitoria 
de Extensão (PROEX). E por fim, dentre os participantes da UFPI, 80\% atuam na Pró-Reitoria de Assuntos Estudantis e Comunitários/PRAEC, sendo especificamente, $70 \%$ por meio do Núcleo de Assistência Estudantil/NAE, e 10\% pelo Núcleo de Acessibilidade/NAU. Outros 20\% estão no Serviço Escola de Psicologia/SEP.

\section{INSTRUMENTOS}

A pesquisa foi realizada com o auxílio dos seguintes instrumentos: Formulário de identificação de Políticas Públicas de atuação do Psicólogo Escolar, visando identificar como o psicólogo atua nas políticas públicas em que está implicado; Roteiro de Entrevista Semiestruturada, versando sobre a Psicologia Escolar na área das Políticas Públicas, com o objetivo de compreender a concepção deles sobre a forma como se delineia o exercício profissional da psicologia nas políticas públicas; Questionário Sociodemográfico, a fim de traçar o perfil de tais profissionais da rede pública de educação; e, Formulário de Registro Documental, com o objetivo de identificar o escopo das políticas educacionais em que se encontram inseridos atuando.

\section{PROCEDIMENTOS}

Submeteu-se a pesquisa ao Comitê de Ética e Pesquisa em Seres Humanos. Após aprovada, iniciou-se a coleta de dados com os participantes de forma voluntária, e assim que receberam explicações acerca do estudo assinaram os Termos de Consentimento Livre e Esclarecidos/TCLE, oficializando a autorização de sua participação. A seguir, foram realizadas as entrevistas semiestruturadas, durante as quais aplicou-se o Formulário de Identificação de Políticas Públicas de Atuação do Psicólogo Escolar, o Roteiro de Entrevista Semiestruturada relativo à interface entre Psicologia Escolar e Políticas Públicas e o Questionário Sociodemográfico. 


\section{ANALISE DE DADOS}

Os dados coletados para esta pesquisa foram analisados por meio da técnica de Análise de Conteúdo, que, segundo Bardin (1977), consiste em técnicas de análise de comunicação que objetiva a compreensão crítica do sentido, conteúdo e as possíveis significações explicitas ou não na comunicação. E, a partir disso, os resultados foram interpretados à luz da Psicologia Escolar Crítica.

\section{RESULTADOS E DISCUSSÃO}

A partir das análises construídas ao longo do processo de investigação, visando contemplar o objetivo deste estudo de identificar e georreferenciar a inserção do psicólogo escolar em instituições públicas de ensino - de âmbito municipal, estadual e federal - junto às políticas públicas educacionais no estado do Piauí, para a discussão dos resultados, tomou-se como referência os seguintes organização: georreferenciamento dos psicólogos atuantes em instituições de ensino no estado por territórios de desenvolvimento e os tipos de Políticas Públicas Educacionais que os profissionais da psicologia têm inserido na atuação.

O Piauí é um estado brasileiro localizado na região nordeste do país, possui 224 municípios organizados em 12 territórios de desenvolvimento. A leitura da realidade do estado a partir da divisão em territórios de desenvolvimento possibilita que se analise criticamente os municípios para que se tenha maior eficácia no planejamento e identificação das especificidades de limitações e potências dos territórios. Mediante esse estudo pode-se constatar a presença do PEE em todos os 12 territórios de desenvolvimento (ver Figura 1.). Entretanto, percebe-se uma maior concentração nos seguintes territórios: Entre Rios e Planície Litorânea - com destaque para o primeiro, no qual se encontra a capital do estado, Teresina. 
Figura 1: Georreferenciamento da/o Psicóloga/o Escolar pelos Territórios de Desenvolvimento do Piaui.

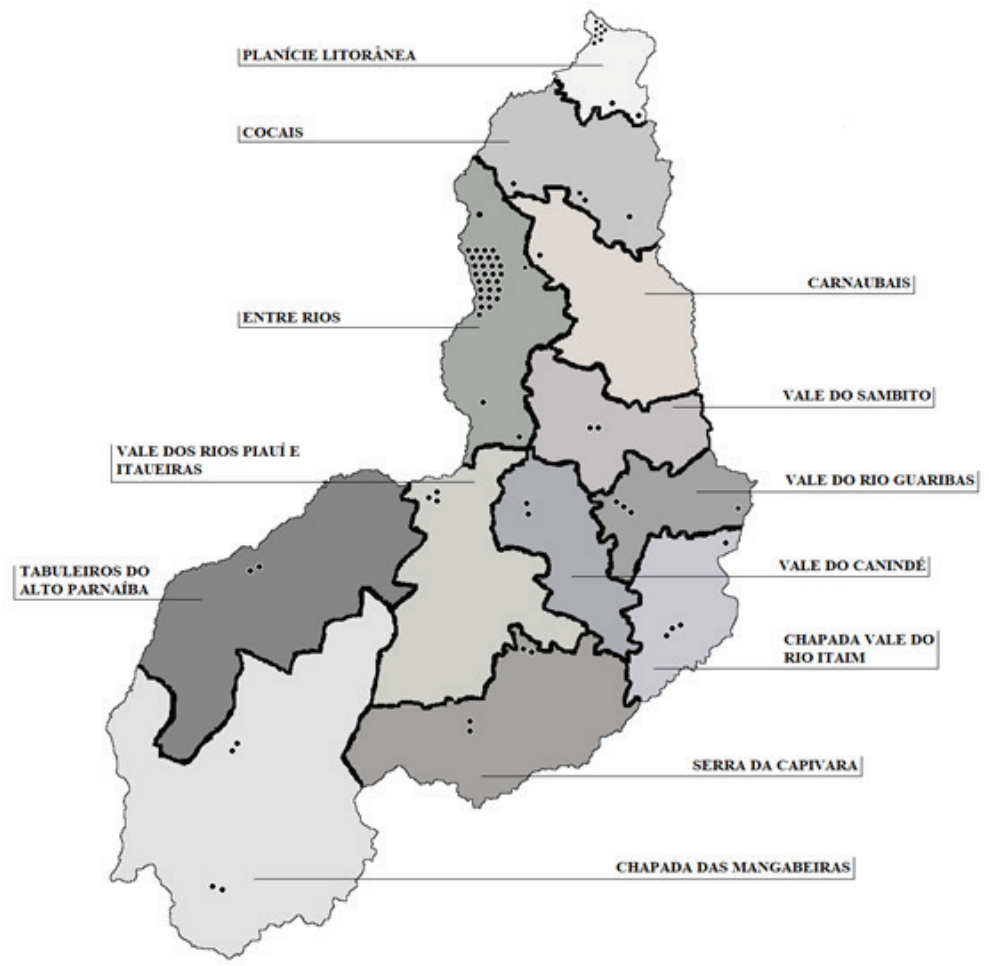

Fonte: Elaborado pelas autoras e pelo autor, 2020.

No que diz respeito à atuação da/do psicóloga/o escolar em Políticas Públicas Educacionais, a partir do estudo pôde-se mapear em quais demandas e finalidades dos tipos de políticas eles estão inseridos, pautando-se nos seis eixos de atuação em políticas públicas listados pelo Grupo de Trabalho (GT) Psicologia e Políticas Educacionais da Associação Nacional de Pesquisa e Pós-graduação em Psicologia (Anpepp), no XVI Simpósio de Pesquisa e Intercâmbio Científico, em junho de 2016 na cidade de Maceió, Alagoas (URT, 2017). 
Tais eixos são: 1) Políticas para educação inclusiva; 2) Políticas de formação e atuação do psicólogo escolar; 3) Políticas de melhoria da educação; 4) Políticas de formação de professores; 5) Políticas de atendimento à diversidade; e 6) Políticas intersetoriais. (URT, 2017).

Assim sendo, segue adiante o Quadro 1, que apresenta o quantitativo de profissionais da psicologia inseridos na atuação em políticas públicas educacionais, por eixo temático. Para a construção e organização dessas informações, levou-se em consideração o tipo de política educacional que as/os psicólogas/ os têm realizado prioritariamente suas atividades profissinais nas suas respectivas instituições públicas de ensino.

\section{Quadro 1- Eixo temático de Políticas Públicas Educacionais e quantitativo de profissionais da psicologia inseridos na atuação.}

\begin{tabular}{|c|l|c|c|}
\hline $\mathrm{N}^{\circ}$ & \multicolumn{1}{|c|}{ Eixo } & $\begin{array}{c}\text { Número de } \\
\text { psicólogas/ } \\
\text { os }\end{array}$ & $\%$ \\
\hline 1 & Políticas para educação inclusiva & 11 & 15,6 \\
\hline 2 & $\begin{array}{l}\text { Políticas de formação e atuação do psicó- } \\
\text { logo escolar }\end{array}$ & 3 & 4,7 \\
\hline 3 & Políticas de melhoria da educação & 44 & 60,9 \\
\hline 4 & Políticas de formação de professores & 7 & 9,4 \\
\hline 5 & Políticas de atendimento à diversidade & 2 & 3,1 \\
\hline 6 & Políticas intersetoriais & 5 & 6,3 \\
\hline
\end{tabular}

Fonte: Elaborado pelas autoras e pelo autor, 2020.

Com base no levantamento realizado junto aos psicólogos participantes da pesquisa, pode-se constatar que 60,9\% dos participantes vinculam práticas junto ao eixo 3 e, portanto, atuam em Políticas voltadas para a melhoria da qualidade da educação em diversos aspectos, tais como o acesso à educação e permanência, manejo dos problemas referentes ao processo de escolarização. 
Dentre as ações que explicitam a prática em Melhorias da Educação, o trabalho em conjunto com a comunidade escolar aparece como característica geral tanto no contexto da Educação Básica, quanto dos IFPIs e UFPIs. Como exemplo, a atuação em políticas educacionais que discutem o acesso e permanência, a organização e estruturação do sistema de ensino, incluindo-se o tempo previsto à educação obrigatória, infraestrutura da escola entre outros.

Entre as diferentes políticas inerentes a esse eixo, destacaram-se as de profissionalização do estudante, por meio do Programa Nacional de Acesso ao Ensino Técnico e ao Emprego/Pronatec, entre alguns psicólogos vinculados às Gerências Regionais de Educação do estado, bem como as políticas de assistência estudantil, referenciadas no Plano Nacional de Assistência Estudantil (Pnaes) apoia a permanência de estudantes de baixa renda matriculados em cursos de graduação presencial das Instituições Federais de Ensino Superior (Ifes).

Não obstante, é imperioso mencionar que além de a inserção e atuação dos psicólogos escolares em políticas de melhoria da educação ter prevalecido no Piauí, foi o único perfil de política educacional identificada nos 12 territórios de desenvolvimento do estado.

Já o eixo 1, Políticas para a Educação Inclusiva, englobou cerca de 15,6\% dos participantes. A inserção de psicólogos escolares nesse eixo envolve a política de inclusão escolar, geral ou de algum segmento específico (por exemplo, surdos, altas habilidades e superdotação, deficiência intelectual, Transtornos Globais de Desenvolvimento - TGDs -, o ensino especial, geral ou de alguma especialidade, bem como atendimentos educacionais para estas pessoas e suas famílias.

Dentre os territórios de desenvolvimento do Piauí que tiveram maior concentração de psicólogos inseridos em políticas para a educação inclusiva, estão o de Entre Rios e Planície Litorânea, regiões onde se encontram as duas cidades com maior 
Produto Interno Bruto/PIB, Teresina, capital piauiense, com R\$ 11 bilhões de PIB, e Parnaíba, com R\$ 1,04 bilhão, além de serem os maiores polos educacionais dessa unidade federativa. (Fundação CEPRO, 2020).

Para mais, é importante destacar que dentre os participantes há apenas um psicólogo que atua em setor destinado especificamente, à garantia de acessibilidade pedagógica, ambiental e aos processos de ensino e aprendizagem de pessoas com deficiência.

Ademais, outras políticas públicas de educação nas quais os psicólogos escolares se apoiam para atuar e foram assim contempladas: 9,4\% em Políticas de Formação de Professores - inclui políticas e programas educacionais que tem como escopo a formação inicial e/ou continuada de professores; $6,3 \% \mathrm{em}$ Políticas Intersetoriais - compreende as políticas que estabelecem interfaces entre a saúde, a educação e a assistência social. Entre estas destacam-se políticas que tratam da saúde dos profissionais da educação, escolarização da infância em abrigos, adolescentes em conflito com a lei (socioeducação), educação em contextos domiciliar, hospitalar e prisional.

Outras políticas públicas em que os psicólogos escolares se amparam para atuar: 4,7\% em Políticas de Formação e Atuação do Psicólogo Escolar - integra as políticas de formação, inserção e atuação de psicólogos escolares nos diversos níveis de educação. Por último, com 3,1\% no eixo das Políticas de Atendimento à Diversidade -, abrange as políticas de direitos humanos, educação no campo, na floresta, dos povos quilombolas, etnias indígenas, populações afrodescendentes e LGBT e Educação de Jovens e Adultos, cotas nas universidades. Dessa maneira, um aspecto bastante relevante a ser discutido sobre a questão do envolvimento - ainda insipiente dos psicólogos do estado com políticas de formação de professores, de políticas intersetoriais e de atendimento à diversidade que esteve presente nos resultados do estudo -, remete à urgência de discussões e ações de inserção da psicologia escolar alicerçadas nas especificidades 
de cada contexto educacional, seja na educação básica, técnico-profissionalizante ou superior.

Muitos psicólogos escolares participantes da pesquisa relataram como lacunas as demandas relacionadas às referidas políticas na formação acadêmica para uma atuação em Psicologia Escolar mais crítica, contextualizada e socialmente referenciada com tais demandas educacionais. Neste aspecto, Freitas (2017) ressalta a importância do processo contínuo de formação do psicólogo escolar visando o fortalecimento da identidade profissional e construção de atuações reflexivas, críticas e éticas.

\section{CONSIDERAÇÕES FINAIS}

A realidade do contexto piauiense se apresenta junto a vários desafios no que diz respeito aos indicadores sociais de desenvolvimento humano e questões educacionais. Neste aspecto, reforça-se a importância de o desempenho profissional do psicólogo escolar estar aliado a uma compreensão acerca da sua participação política no âmbito educacional, assim como no que se refere a ações direcionadas à melhoria da educação pública ofertada no estado por meio do fortalecimento de práticas educacionais coletivas e institucionais.

Dentre as dificuldades da inserção dessa atuação profissional de forma ampla no Piauí destaca-se a centralização da oferta do serviço em regiões específicas - em especial aquelas mais abastadas -, sendo necessário um movimento de expansão da atuação da psicologia escolar pelas demais regiões do estado. Vê-se, pois, que há urgência na implementação de diretrizes institucionais e políticas que impulsionem a inserção de profissionais da área referida junto à rede pública de educação.

E, acima de tudo, sem perder de vista as formas de desdobramentos das políticas públicas de educação diante das potencialidades histórico-culturais, econômicas e sociais dos diferentes territórios de desenvolvimento do estado do Piauí, que estarão mediados pela inserção desses profissionais, atendendo 
à operacionalização da recente aprovação da Lei nº13.935, em dezembro de 2019, de âmbito nacional, que implementa a prestação de serviços de psicologia e serviço social na rede pública de educação básica foi uma grande conquista e avanço no que se refere a dificuldade supracitada.

Ademais, há algumas dificuldades próprias da atuação na escola que são produzidas pela mobilização de competências teórico-práticas e transversais. Assim, faz-se necessário que a formação inicial e continuada seja aliada para atenuar essas dificuldades. O entendimento desses aspectos deve ser tensionado, pautando-se inclusive na ampliação da discussão acerca da atuação psicossocial e política do psicólogo escolar de forma crítica e participativa.

Apesar disso, mesmo com o significativo destaque da inserção de profissionais da psicologia em políticas educacionais de melhoria da educação, seguido das políticas para a educação inclusiva no estado do Piauí, ainda se encontra de forma incipiente e introdutória a sua integração em políticas de formação de professores, políticas intersetoriais e em políticas de atendimento à diversidade.

Tais aspectos podem ser pensados e desdobrados em aproximações entre as instituições formadoras de psicólogos, as gerências e secretarias educacionais, os movimentos sociais e os poderes Executivo e Legislativo municipais e estadual, ao desdobrarem planejamentos concatenados com a luta pelo direito à educação e pelo acesso à escola, sobretudo para uma parcela significativa da população do estado, que, historicamente lhe foram negados.

Dessa forma, mediante este estudo foi possível fazer um mapeamento das psicólogas e psicólogos escolares inseridos em Políticas Públicas de Educação no Piauí, identificando os eixos de políticas nos quais estão inseridos. Esta investigação incentivou ainda reflexões sobre possíveis limitações e potencialidades características da realidade do contexto, gerando possibilidades 
para a ampliação e fortalecimento da visão acerca da função social da Psicologia Escolar Crítica, bem como, a atuação frente às Políticas Públicas Educacionais.

\section{REFERÊNCIAS}

BARDIN, L. Análise de conteúdo. (L. de A. Rego \& A. Pinheiro, Trads.). Lisboa: Edições 70, 2006. (Obra original publicada em 1977).

CARVALHO, D. B. O trabalho docente em psicólogo e o enfrentamento da violência contra crianças e adolescentes: uma experiência teresinense. 2007. 318 f. Tese (Doutorado em Psicologia Social; Processos Psicossociais; Relações de Poder e Sociedade) - Universidade Federal do Rio Grande do Norte, Natal, 2007.

COLLARES-DA-ROCHA, J. C. C.; OLIVEIRA, D. R. A participação política do psicólogo escolar: modos e ações. In: NEGREIROS, F.; MAIA, J. B. D. (Org.). Psicologia escolar, atuação político-legislativa e luta antimedicalizante. (p. 43-53). Curitiba: CRV, 2020.

CRUZ, L. R.; GUARESCHI, N. Políticas públicas e assistência social: Diálogo com práticas psicológicas. Editora Vozes Limitada, 2017.

DAMASCENO, M. A.; NEGREIROS, F. Professores, fracasso e sucesso escolar: um estudo no contexto educacional brasileiro. Revista de Psicologia da IMED, v. 10, n. 1, p. 73-89, 2018. Disponível em: https://seer.imed.edu. br/index.php/revistapsico/article/view/2572. Acesso: 10 nov. 2020.

DIMENSTEIN, M. A ação clínica e os espaços institucionais das políticas públicas: desafios éticos e técnicos. In: Conselho Federal de Psicologia. V seminário nacional psicologia e políticas públicas: subjetividade, cidadania e políticas públicas: subjetividade, cidadania e políticas públicas (p. 119-126). Brasília: CFP, 2011.

FARAH, M. F. S. Análise de políticas públicas no Brasil: de uma prática não nomeada à institucionalização do" campo de públicas". Revista de Administração Pública, v. 50, n. 6, p. 959-979, 2016. DOI: https://doi. org/10.1590/0034-7612150981. Acesso: 10 nov. 2020..

FERNANDES, A. M. D.; MÉLO, S. C. B. Formação e gestão na escola: atravessamentos das políticas educacionais na ação da psicologia escolar. Comissão de Psicologia e Educação (Org.), Conversações em Psicologia e Educação, p. 151-160, 2016. 
FOUCAULT, M. Os anormais. São Paulo: Martins Fontes, 2010.

FUNDAÇÃO CEPRO - Fundação Centro de Pesquisas Econômicas e Sociais do Piauí. Piauí em números. 2020. Disponível em: http://www.cepro.pi.gov. br/index.php. Acesso: 10 nov. 2020.

FREITAS, R. A. D. (2017). Formação continuada com psicólogos escolares da Ceilândia/DF: potencialidades da pesquisa-intervenção. (Dissertação de mestrado não-publicada). Universidade de Brasília, Brasília.

GIL, A. C. Como elaborar projetos de pesquisa. São Paulo: Atlas, 2010.

GUZZO, R. S. L.; COSTA, A. S. C.; SANT’ANA, I. M. Formando psicólogos escolares: problemas, vulnerabilidade desafios e horizontes. In: MARINHO-ARAÚJO, C. M. (Org.). Psicologia escolar: novos cenários e contextos de pesquisa, prática e formação. Campinas: Alínea, 2009. p. 16-35.

LEONARDO, N. S. T.; ROSSATO, S. P. M.; CONSTANTINO, E. P. M. Políticas públicas em educação e o fracasso escolar: Interlocuções com a Psicologia. In CAMPOS, H.; SOUZA, M. P. R.; FACCI, M. G. D. Psicologia e políticas educacionais. Curitiba: Appris, 2018.

LESSA, S.; TONET, I. Capital e Estado de Bem-estar. São Paulo: Instituto Lukács, 2013.

MACEDO, J. P.; DIMENSTEIN, M. Formação do psicólogo para a saúde mental: a psicologia piauiense em análise. Interface-Comunicação, Saúde, Educação, v. 15, n. 39, p. 1145-1158, 2011. DOI: https://doi.org/10.1590/ S1414-32832011005000021.

MANSANO, Sonia Regina Vargas. Sorria, você está sendo controlado: resistência e poder na sociedade de controle. São Paulo: Summus, 2009.

MORAES, C. M.; CARVALHO, L. S.; COSTA, T. S.; NEGREIROS, F.; FREIRE, S. E. A. Políticas educacionais e Psicologia: Uma revisão da literatura. Revista Psicologia-Teoria e Prática, v. 21, n. 3, 2019. Disponível em: http:// editorarevistas.mackenzie.br/index.php/ptp/article/view/11814

NEGREIROS, F.; CAMPOS, H. R. (2020). Entrevista com Prof. Dr. Herculano Ricardo Campos. Psicologia Escolar e Educacional, v. 24, 2020. DOI: https://doi.org/10.1590/2175-35392020234423 
ROMAGNOLI, R. C. Algumas reflexões acerca da clínica social. Revista do Departamento de Psicologia, v. 18, n. 2, p. 47-56, 2006. DOI: https://doi. org/10.1590/S0104-80232006000200004

SANTOS, D. Educação e precarização profissionalizante: crítica à integração da escola com o mercado. São Paulo: Instituto Lukács, p. 190-209, 2017.

SILVA, C. A.; NEGREIROS, F. A formação do psicólogo no Piauí: caracterização curricular e perfil do futuro docente. In S. E. Moraes (Org.), Currículo e formação docente: um diálogo interdisciplinar (pp. 423-439). Campinas, SP: Mercado de Letras, 2008.

SILVA, C. A.; YAMAMOTO, O. H. As políticas sociais na formação graduada do psicólogo no Piauí. Psicologia: ciência e profissão, v. 33, n. 4, p. 824-839, 2013. DOI: http://dx.doi.org/10.1590/S1414-98932013000400005.

SILVA, R. B.; CARVALHAES, F. F. Psicologia e políticas públicas: impasses e reinvenções. Psicologia \& Sociedade, v. 28, n. 2, p. 247-256, 2016. DOI: https://doi.org/10.1590/1807-03102016v28n2p247

SOUZA, M. P. R.; CUNHA, B. B. B. Projetos de Lei e políticas públicas: o que a psicologia tem a propor para a Educação. In: ANGELUCCI, C.; SOUZA, B. P. Medicalização de crianças e adolescentes: conflitos silenciados pela redução de questões sociais a doenças de indivíduos. São Paulo: Casa do Psicólogo, p. 71-110, 2010.

SOUZA, M. P. R.; RAMOS, J. M.; LIMA, C. P.; BARBOSA, D. R.; CALADO, V. A.; YAMAMOTO, K. Atuação do psicólogo na educação: análise de publicações científicas brasileiras. Psicologia da Educação, n. 38, p. 123-138, 2014. Disponível em: https://revistas.pucsp.br/psicoeduca/article/view/22808. Acesso: 10 nov. 2020.

TURATO, Egberto Ribeiro. Métodos qualitativos e quantitativos na área da saúde: definições, diferenças e seus objetos de pesquisa. Revista de Saúde pública, v. 39, p. 507-514, 2005. DOI: https://doi.org/10.1590/S003489102005000300025

URT, S. C. Políticas educacionais e formação: produção, projetos e ações em psicologia. Campo Grande: Editora Oeste, 2017.

ZIBETTTI, M. L. T.; PACÍFICO, J. M.; TAMBORIL, M. I. B. A educação como direito: considerações sobre políticas educacionais. In: CAMPOS, H. R.; SOUZA, M. P. R.; FACCI, M. G. D. (org.). Psicologia e políticas educacionais (pp. 17-42). Curitiba: Appris, 2018. 
Autores:

Fauston Negreiros (e-mail: faustonnegreiros@ufpi.edu.br)

Rafaela Brenda Araújo da Silva (e-mail: rafisilva0@gmail.com)

Janaina Oliveira Rocha (e-mail: janainaa_rochaa@hotmail.com)

Thaisa da Silva Fonseca (e-mail: thaisafonseca23@hotmail.com)

Leilanir de Sousa Carvalho (e-mail: leilanircarvalho@ymail.com)

Fernanda Maria de Oliveira (e-mail: fmariaoli@yahoo.com.br) 